\title{
Maximizing the Route Capacity in Cognitive Radio Networks
}

\author{
Angela Sara Cacciapuoti, Marcello Caleffi, Francesco Marino, Luigi Paura \\ Department of Electrical Engineering and Information Technologies (DIETI) \\ University of Naples Federico II, Italy \\ Email:\{angelasara.cacciapuoti, marcello.caleffi, francesco.marino, paura\}@unina.it
}

\begin{abstract}
The problem of choosing the route providing the best communication opportunities among the available routes is particularly challenging in self-organizing Cognitive Radio networks, since the communication opportunities are deeply affected by the primary-user (PU) activity. Furthermore, whenever the route selection exploits proactively acquired information on the PU activity, routing update packets need to be periodically exchanged among the nodes. The time interval between these exchanges, i.e., the routing update period, deeply affects the overall communication opportunities provided by a route, regardless of the adopted routing protocol. Hence, in this paper, we analytically derive the optimal route priority rule in the sense of maximizing the average capacity, by accounting for both the PU activity and the routing update period. The theoretical analysis is conducted by adopting two different widely-adopted PU activity models to confer generality to the analysis. Finally, the analytical results are validated through numerical evaluations.
\end{abstract}

Index Terms-Cognitive Radio; routing; update.

\section{INTRODUCTION}

Cognitive Radio (CR) paradigm has been recognized as a viable solution for the deployment of spectrum-efficient selforganizing networks [1], [2] within the Smart City concept, since it combines cross-layer optimization and learning mechanisms. In particular, CR paradigm counteracts both spectrum inefficiency and spectrum scarcity by allowing unlicensed users, referred to as CR users, to exploit the spectrum holes, i.e., portions of the radio spectrum temporarily vacated by licensed users, referred to as Primary Users (PUs), for establishing multi-hop communications in a peer-to-peer fashion [3]. To fully unleash the potentials of the CR paradigm, new challenges must be addressed and solved at the network layer.

In particular, let us consider a typical routing problem, i.e., a source node must select, among the available routes, the one providing the best communication opportunities to forward the packets toward the destination node. Each route generally exhibits specific communication characteristics, such as capacity, route failure probability, end-to-end delay, etc.. Hence, the criteria for establishing a priority among the available routes, i.e., for choosing the optimal route, deeply affects the routing performance, regardless of the adopted routing protocol [4], [5].

The aforementioned route priority issue is even more crucial

This work was partially supported by the Italian government under PON0102425 "SIRIO: Services for wIreless netwoRk Infrastructure beyOnd 3G".

978-1-4799-4657-0/14/\$31.00 (c) 2014 IEEE in $\mathrm{CR}$ networks, due to effects induced by the PU activities over the route availability [6]. In fact, the more persistent is the PU activity on a route, the shorter is the time interval during which the route is available to the CR network. Consequently, although a route, say route $r_{m}$, could exhibit more appealing characteristics (i.e., higher capacity) with respect to another route, say route $r_{l}$, in absence of PU activity, the presence of PU activity can make $r_{l}$ a better choice than $r_{m}$, i.e., the route priority depends also on the PU activity.

Furthermore, whenever the source selects the route basing on proactively acquired information on the PU activity, routing update packets need to be periodically exchanged among the nodes. The time interval between these exchanges, referred to as routing update period, deeply affects the overall routing performance, independently of the adopted routing protocol. In fact, the shorter are the update periods, the more accurate are the the routing decisions [7]. However, the shorter are the periods, the higher is the overhead induced within the network.

Besides the overall routing performance, the routing update period affects the optimal priority among the routes. Whenever the source receives a routing update, it acquires some knowledge on the current PU activity over the different routes. Clearly, the shorter are the update periods, the more the source can exploit such a knowledge to prioritize the available routes.

Hence, in this paper, we analytically derive the optimal route priority rule in the sense of maximizing the capacity available at the arbitrary CR source, by accounting for both the PU activity and the routing update period. Specifically, at first, the problem of the optimal route priority rule is formulated by jointly accounting for both the PU activities and the routing update period. Such a formulation takes also into account the slotted nature of the CR time induced by the spectrum sensing functionality [8]. Then, we analytically derive the optimal route priority rule, and the theoretical analysis is carried out by adopting two different widely-adopted PU activity models [7] for conferring generality to the analysis: i) Bernoulli PU Activity Model, in which the PU activity is time independent; ii) Markov PU Activity Model, in which the PU activity exhibits a time correlation according to a Markov Chain.

The rest of the paper is organized as follows. In Sec. II we describe the network model and we collect some definitions that will be used through the paper. In Sec. III we derive the optimal route priority rule and we analytically show that it maximizes the available capacity at the CR source node. We 


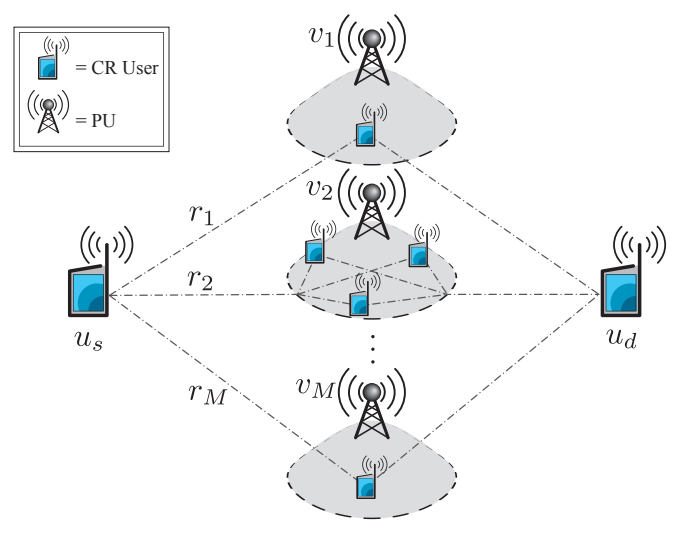

Fig. 1. Network Model.

evaluate the analytical results numerically in Sec. IV. Finally, in Sec. V we conclude the paper.

\section{Network Model And Preliminaries}

In this section, we first describe the network model. Then, we define the terms used through the paper.

\section{A. Network Model}

The CR user time is organized into fixed-sized slots of duration $T$. Each time slot $T$ is further organized in a sensing period $T_{s}$, which measures the portion of the time slot assigned to the spectrum sensing, and in a transmission period $T_{t x}$, which measures the portion of the time slot devoted to the CR packet transmissions.

The adopted network model is shown in Fig. 1. Specifically, an arbitrary CR source, say $u_{s}$, can communicate with an arbitrary CR destination, say $u_{d}$, through $M$ different routes $\left\{r_{m}\right\}_{m=1}^{M}$. The arbitrary route $r_{m}$ is affected ${ }^{1}$ by the activity of PU $v_{m}$, and the PU activities on different routes are assumed independent ${ }^{2}$.

With reference to a generic route $r_{m}$, two different widelyadopted PU activity models are considered to confer generality to the analysis.

1) Bernoulli PU Activity Model: The activity of PU $v_{m}$ is modeled as a Bernoulli process. Specifically, the PU activity is assumed independent and identically distributed among different time slots of duration $T$. In each time slot, $v_{m}$ is inactive with probability $p_{\text {off }}^{m}$ and active with probability $p_{\mathrm{on}}^{m}=1-p_{\mathrm{off}}^{m}$.

2) Markov PU Activity Model: The activity of PU $v_{m}$ on the arbitrary $n$-th time slot $X_{m}(n)$ is modeled as a two-state Markov process, hence the PU activity in subsequent time slots is correlated. In the on state, denoted in the following as state $1, v_{m}$ is active with probability $p_{\mathrm{on}}^{m}$ whereas in the off

\footnotetext{
${ }^{1}$ When multiple PUs affect the same route $r_{m}, v_{m}$ models the aggregate PU activity induced by multiple PUs, with $v_{m}$ being active whenever any of the multiple PUs is active.

${ }^{2}$ When the PU activities on different routes are correlated, e.g., a single PU affects two adjacent routes, either $r_{m}$ represents the highest-capacity route belonging to a set of correlated routes or $C_{m}$ represents the cumulative capacity of the set of correlated routes.
}

state, denoted in the following as state 0 , it is inactive with probability $p_{\mathrm{off}}^{m}=1-p_{\mathrm{on}}^{m}$. The distribution of the on-off periods determines the expressions of the transition probabilities [9], [10]. Specifically, if the on-off periods are exponentially distributed with parameters $\lambda_{1}^{m}$ and $\lambda_{0}^{m}$, the transition probabilities are given by: $p_{0 \mid 1}^{m} \triangleq P\left(X_{m}(n+1)=0 \mid X_{m}(n)=\right.$ $1)=p_{\text {off }}^{m}+p_{\text {off }}^{m} e^{-\left(\lambda_{1}^{m}+\lambda_{0}^{m}\right) T}, p_{1 \mid 1}^{m}=1-p_{0 \mid 1}^{m}, p_{0 \mid 0}^{m} \triangleq$ $P\left(X_{m}(n+1)=0 \mid X_{m}(n)=0\right)=p_{\text {off }}^{m}+p_{\text {on }}^{m} e^{-\left(\lambda_{1}^{m}+\lambda_{0}^{m}\right) T}$ and $p_{1 \mid 0}^{m}=1-p_{0 \mid 0}^{m}$. By accounting for the Markov chain property, we have $p_{\text {off }}^{m}=p_{0 \mid 1}^{m} /\left(p_{0 \mid 1}^{m}+p_{1 \mid 0}^{m}\right), p_{\mathrm{on}}^{m}=p_{1 \mid 0}^{m} /\left(p_{0 \mid 1}^{m}+p_{1 \mid 0}^{m}\right)$.

\section{B. Definitions and Assumptions}

Definition 1. (Routing Update Period) The routing update period $K T$ is the time interval between the reception of two route update packets.

Remark. The routing update period being a multiple of the time slot $T$ allows us to account for the slotted nature of the CR time induced by the spectrum sensing functionality.

Assumption 1. (Allowed Route) With reference to an arbitrary routing update period ${ }^{3}$, the CR source $u_{s}$ can select the route $r_{m}$ for packet transmission during an arbitrary time slot if and only if, within the previous routing update packet, the route $r_{m}$ was reported as available during the time slot in which the update packet was received.

Remark. Assumption 1 is not restrictive: the results derived in the following can be easily extended to the case of a routing update received at time slot $\tilde{n}$ and reporting the route availability during a previous time slot. Furthermore, considering as allowed only the routes reported as available during the routing update slot is reasonable in smart cities scenarios, generally characterized by delay-sensitive requirements.

Definition 2. (Route Set) The route set $\mathcal{R}$ is an ordered sequence of routes $\left\{r_{m}\right\}_{m=1}^{M}$ :

$\mathcal{R}=\left(r_{k_{1}}, r_{k_{2}}, \ldots, r_{k_{M}}\right), k_{m} \neq k_{l} \forall m \neq l \wedge k_{m} \in\{1, \ldots, M\}$

Assumption 2. (Route Priority) Given the route set $\mathcal{R}$ defined in (1), route $r_{k_{m}}$ is used by the CR source $u_{s}$ during the arbitrary time slot $n$ if and only if: i) $r_{k_{m}}$ is an allowed route within the current routing update period; ii) any other route $r_{k_{l}}$, with $k_{l}<k_{m}$, is not allowed within the current routing update period.

Remark. Through Assumption 2, we introduce an order relation over the route set. Specifically, the order reflects the priority of the different routes within a routing update period. Fig. 2 shows an example of the route priority over the time domain, when $M=3$. In the figure, we report the PU activity states for each time slot, along with the route set $\mathcal{R}$. More in detail, during the first routing update period the source selects route $r_{1}$, i.e., $r_{k_{1}}=r_{1}$ in (1), since: i) $r_{1}$ is the route with

\footnotetext{
${ }^{3}$ In the following, we omit the routing update period dependence from the notation of the allowed route for the sake of simplicity.
} 
highest priority; ii) the routing update packet reports the PU state on $r_{1}$ as off in the first time slot. During the second routing update period, the source selects route $r_{2}$ instead of $r_{1}$, i.e., $r_{k_{1}}=r_{2}$ since: i) the route with highest priority $r_{1}$ is not available; ii) the routing update packet reports the PU state on $r_{2}$ as off in the first time slot.

Definition 3. (Route Overhead) Given the route $r_{m}$, the route overhead $\Omega_{m}(K)$ is the average bit-rate needed to propagate the routing information through $r_{m}$ :

$$
\Omega_{m}(K)=\frac{L_{m}}{K T}
$$

where $L_{m}$ is the bit cost associated with the reception of the routing update packet at the CR source $u_{s}$ and $K$ is the routing update parameter.

Definition 4. (Route Capacity) Given the route $r_{m}$, the route capacity $C_{m}$ is the average bit-rate achievable by the $\mathrm{CR}$ source $u_{s}$ in an arbitrary time slot when: i) no PU activity is present on route $r_{m}$ during the time slot; ii) no routing updates packet are propagated through route $r_{m}$ during the time slot.

Remark. With reference to the example depicted in Fig. 2, we have that, since in the second, third and sixth time slot of the first routing update period PU $v_{1}$ is on, the available capacity in such time slots is defined as zero. We note that this assumption is not restrictive, since the formulas derived in the following can be easily extended to case of a not-null capacity during an arbitrary time slot when the PU is active, and the derived results continue to hold.

Definition 5. (Average Aggregate Route Capacity) Given the arbitrary route set $\mathcal{R}$ and the routing update parameter $K$, the average aggregate route capacity $\bar{C}_{\mathcal{R}}(K)$ is the average capacity available at the CR source $u_{i}$ by accounting for both the PU activities on the $M$ routes and the route overhead $\Omega_{m}(K)$ induced by the update packets.

Definition 6. (Optimal Priority Rule) Given the routing update parameter $K$, the Optimal Priority Rule is a criterium for ordering the routes in the route set $\mathcal{R}$ that allows to maximize the average aggregate route capacity for a given routing update period $K$.

\section{Optimal Route PRIORITY}

In this section we derive the optimal route priority rule maximizing the average aggregate capacity $\bar{C}_{\mathcal{R}}(K)$ for a given routing update period $K$ (Theorem 1). To this aim, we need the intermediate results stated in Propositions 1-3.

Proposition 1. (Average Route Capacity: Bernoulli PU Activity Model) Given the routing update parameter K, when the Bernoulli PU activity model is adopted, the average route capacity $\bar{C}_{r_{m}}^{\mathrm{BE}}(K)$ provided by the allowed route $r_{m}$ is:

$$
\begin{aligned}
\bar{C}_{r_{m}}^{\mathrm{BE}}(K) & =C_{m}\left[1+(K-1) p_{\text {off }}^{m}\right]-\Omega_{m}(K) \\
& \triangleq \bar{\Psi}_{r_{m}}^{\mathrm{BE}}(K)-\Omega_{m}(K)
\end{aligned}
$$

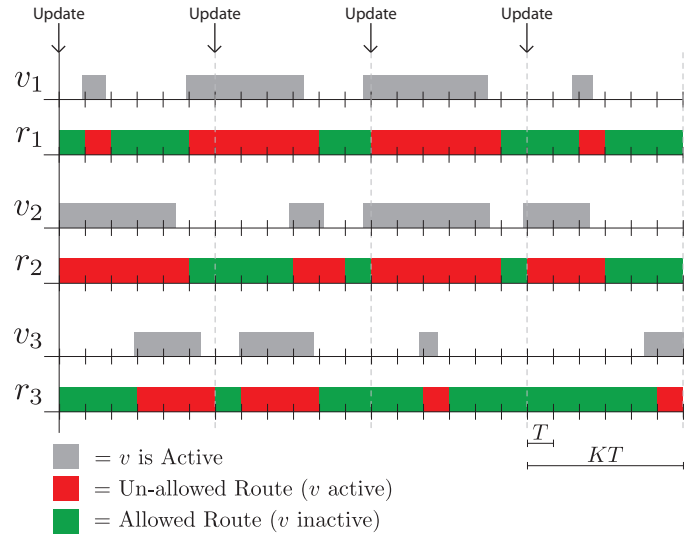

Fig. 2. Routing Update Strategy.

where $\Omega_{m}(K)$ is defined in (2).

Proof: Reported in [11].

Remark. Physically, within our model we assume: i) the arbitrary route $r_{m}$ is affected by some PU activity; ii) the CR source $u_{s}$ is not directly affected by such a PU activity, i.e., it acquires knowledge on such activity only through the routing update packets. Furthermore, we assume that only allowed routes, i.e., routes available during the time slot in which the routing update packet is received, can be used within the routing update period (Assumption 1).

Remark. The average route capacity $\bar{C}_{r_{m}}^{\mathrm{BE}}(K)$ provided by the allowed route $r_{m}$ is function of four terms: i) the off state probability $p_{\text {off }}^{m}$ of PU $v_{m}$; ii) the route capacity $C_{m}$; iii) the routing update period $K$; i) the corresponding route overhead $\Omega_{m}(K)$.

Proposition 2. (Average Route Capacity: Markov Chain PU Activity Model) Given the routing update parameter $K$, when the Markov chain PU activity model is adopted, the average route capacity $\bar{C}_{r_{m}}^{\mathrm{MC}}(K)$ provided by the allowed route $r_{m}$ is:

$$
\bar{C}_{r_{m}}^{\mathrm{MC}}(K)=\bar{\Psi}_{r_{m}}^{\mathrm{MC}}(K)-\Omega_{m}(K)
$$

where $\Omega_{m}(K)$ is defined in (2) and $\bar{\Psi}_{r_{m}}^{\mathrm{MC}}(K)$ is given by the following recursive expression:

$$
\begin{aligned}
& \bar{\Psi}_{r_{m}}^{\mathrm{MC}}(K)=K C_{m}-p_{1 \mid 0}^{m}\left[\bar{\Psi}_{r_{m}}^{\mathrm{MC}}(K-1)+\right. \\
& \left.+\sum_{l=1}^{K-2}\left(p_{1 \mid 1}^{m}\right)^{l} \bar{\Psi}_{r_{m}}^{\mathrm{MC}}(K-l-1)\right]
\end{aligned}
$$

with $\bar{\Psi}_{r_{m}}^{\mathrm{MC}}(1)=C_{m}$ and $\bar{\Psi}_{r_{m}}^{\mathrm{MC}}(2)=2 C_{m}-p_{1 \mid 0}^{m} C_{m}$.

Proof: Reported in [11].

Remark. Similarly to (3), the average route capacity $\bar{C}_{r_{m}}^{\mathrm{MC}}(K)$ provided by the allowed route $r_{m}$ is function of: i) the off state probability $p_{\text {off }}^{m}$ of PU $v_{m}$; ii) the route capacity $C_{m}$; iii) the routing update period $K$; i) the corresponding route overhead $\Omega_{m}(K)$. Differently from (3), $\bar{C}_{r_{m}}^{\mathrm{MC}}(K)$ depends also on the PU transition probabilities $p_{1 \mid 1}^{m}$ and $p_{1 \mid 0}^{m}$. 
Proposition 3. (Average Aggregate Route Capacity) Given the routing update parameter $K$ and the route set $\mathcal{R}$, the average aggregate route capacity $\bar{C}_{\mathcal{R}}(K)$ available at the source $u_{s}$ is equal to:

$$
\begin{aligned}
\bar{C}_{\mathcal{R}}(K) & =\sum_{m=1}^{M} \bar{C}_{r_{m}}(K) p_{\mathrm{off}}^{m} \prod_{n=1}^{m-1} p_{\mathrm{on}}^{n} \\
& =\sum_{m=1}^{M}\left(\bar{\Psi}_{r_{m}}(K)-\Omega_{m}(K)\right) p_{\mathrm{off}}^{m} \prod_{n=1}^{m-1} p_{\mathrm{on}}^{n}
\end{aligned}
$$

where $\bar{C}_{r_{m}}(K)$ and $\bar{\Psi}_{r_{m}}(K)$ are defined in (3) if the Bernoulli PU Activity Model is adopted, otherwise $\bar{C}_{r_{m}}(K)$ and $\bar{\Psi}_{r_{m}}(K)$ are defined in (4) and (6).

Proof: Reported in [11].

Definition 7. (Aggregate Route Overhead) Given the routing update parameter $K$ and the route set $\mathcal{R}$, the aggregate route overhead $\Omega(K)$ represents the loss in terms of average aggregate capacity due to the overhead induced by the routing update:

$$
\Omega(K)=\sum_{m=1}^{M} \Omega_{m}(K) p_{\text {off }}^{m} \prod_{n=1}^{m-1} p_{\text {on }}^{n}
$$

We can now derive the optimal priority rule.

Rule 1. Let us consider two routes $r_{i}$ and $r_{j}$ that satisfy the following conditions:

$$
\begin{array}{ll}
\text { 1) } & C_{i}-\Omega_{i}(K) \geq C_{j}-\Omega_{j}(K) \\
\text { 2) } & C_{i}\left(p_{0 \mid 0}^{i}\right)^{K-1} \geq C_{j}\left(p_{0 \mid 0}^{j}\right)^{K-1} \\
\text { 3) } & C_{i} p_{1 \mid 0}^{i}\left(p_{1 \mid 1}^{i}\right)^{K-2}<C_{j} p_{1 \mid 0}^{j}\left(p_{1 \mid 1}^{j}\right)^{K-2}
\end{array}
$$

We refer to these three conditions as Rule 1 . When the PU activities are modeled according to the Bernoulli model, clearly condition 2) and 3) collapse in the unique condition $C_{i} p_{\text {off }}^{i} \geq C_{j} p_{\text {off }}^{j}$. Hence Rule 1 becomes:

$$
\begin{array}{ll}
\text { 1) } & C_{i}-\Omega_{i}(K) \geq C_{j}-\Omega_{j}(K) \\
\text { 2) } & C_{i} p_{\text {off }}^{i} \geq C_{j} p_{\text {off }}^{j}
\end{array}
$$

Proposition 4. Given the routing update parameter $K$ the average route capacity $\bar{C}_{r_{m}}(K)$ provided by route $r_{m}$ is greater than the average route capacity $\bar{C}_{r_{l}}(K)$ provided by route $r_{l}$, i.e., $\bar{C}_{r_{m}}(K)>\bar{C}_{r_{l}}(K)$, if and only if route $r_{m}$ satisfies the conditions of Rule 1 with respect to route $r_{l}$.

Proof: Reported in [11].

Theorem 1. Let $(S, D)$ be a generic $C R$ flow. Any route set $\mathcal{R}$ ordered according to Rule 1 , achieves an average aggregate route capacity $\bar{C}_{\mathcal{R}}(K)$ greater than the average aggregate route capacity $\bar{C}_{\mathcal{R}^{\prime}}(K)$ associated with a route set $\mathcal{R}^{\prime}$ composed by the same routes but in a different order:

$$
\bar{C}_{\mathcal{R}}(K)>\bar{C}_{\mathcal{R}^{\prime}}(K)
$$

Proof: Let $\mathcal{R}=\left\{r_{1}, \ldots, r_{i-1}, r_{i}, r_{i+1}, \ldots, r_{M}\right\}$ be the route set ordered according to Rule 1 . Let $\mathcal{R}^{\prime}=$ $\left\{r_{1}, \ldots, r_{i-1}, r_{i+1}, r_{i}, \ldots, r_{M}\right\}$ be the route set constituted

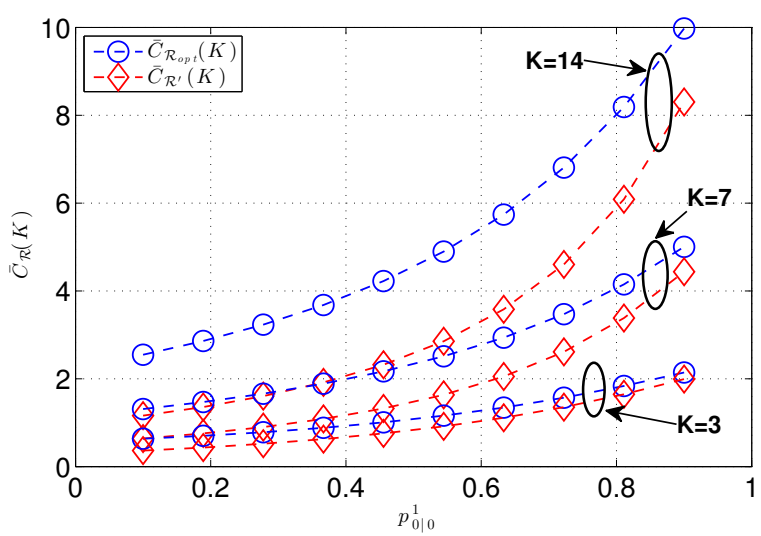

Fig. 3. Average Aggregate Capacity: Markov PU Activity Model.

by the same routes of $\mathcal{R}$ but ordered according to a different criterion. In order to prove the theorem we conduct a reductio ad absurdum, i.e. we suppose

$$
\bar{C}_{\mathcal{R}}(K)<\bar{C}_{\mathcal{R}^{\prime}}(K)
$$

According to the result of Proposition 3, $\bar{C}_{\mathcal{R}}(K)$ and $\bar{C}_{\mathcal{R}^{\prime}}(K)$ can be expressed as respectively:

$$
\begin{aligned}
& \bar{C}_{\mathcal{R}}(K)=\sum_{m=1}^{i-1} \bar{C}_{r_{m}}(K) p_{\mathrm{off}}^{m} \prod_{n=1}^{m-1} p_{\mathrm{on}}^{n}+ \\
& +\bar{C}_{r_{i}}(K) p_{\mathrm{off}}^{i} \prod_{l=1}^{i-1} p_{\mathrm{on}}^{l}+\bar{C}_{r_{i+1}}(K) p_{\mathrm{off}}^{i+1} p_{\mathrm{on}}^{i} \prod_{l=1}^{i-1} p_{\mathrm{on}}^{l}+ \\
& +\sum_{m=i+2}^{M} \bar{C}_{r_{m}}(K) p_{\mathrm{off}}^{m} \prod_{n=1}^{m-1} p_{\mathrm{on}}^{n} \\
& \bar{C}_{\mathcal{R}^{\prime}}(K)=\sum_{m=1}^{i-1} \bar{C}_{r_{m}}(K) p_{\mathrm{off}}^{m} \prod_{n=1}^{m-1} p_{\mathrm{on}}^{n}+ \\
& +\bar{C}_{r_{i}+1}(K) p_{\mathrm{off}}^{i+1} \prod_{l=1}^{i-1} p_{\mathrm{on}}^{l}+\bar{C}_{r_{i}}(K) p_{\mathrm{off}}^{i} p_{\mathrm{on}}^{i+1} \prod_{l=1}^{i-1} p_{\mathrm{on}}^{l}+ \\
& +\sum_{m=i+2}^{M} \bar{C}_{r_{m}}(K) p_{\mathrm{off}}^{m} \prod_{n=1}^{m-1} p_{\mathrm{on}}^{n}
\end{aligned}
$$

By combining (13) and (14) in (12) and by simplifying the common terms, (12) is rewritten as:

$$
\bar{C}_{r_{i}+1}(K)>\bar{C}_{r_{i}}(K)
$$

Hence, $\bar{C}_{\mathcal{R}}(K)<\bar{C}_{\mathcal{R}^{\prime}}(K) \Leftrightarrow \bar{C}_{r_{i}+1}(K)>\bar{C}_{r_{i}}(K)$. This constitutes an absurdum according to the results of Proposition 4.

\section{Performance Analysis}

In this section, we validate the theoretical analysis through numerical evaluation. Specifically, we consider $M=2$ routes, and the parameter setting is as follows: the normalized bit costs $L_{m}$ associated with the update packet are set equal to $L_{1} / T=0.2$ and $L_{2} / T=0.7$, and the route capacities $C_{m}$ 


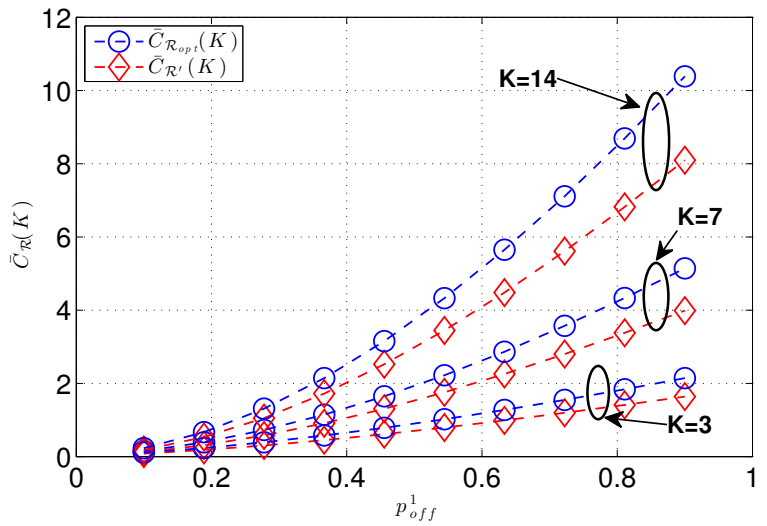

Fig. 4. Average Aggregate Capacity: Bernoulli PU Activity Model.

are set equal to $C_{1}=0.9$ and $C_{2}=1$. Clearly, if we neglect the effects of the routing update overhead, we have that route $r_{2}$ would be preferable over route $r_{1}$.

In Figure 3, we report the average aggregate capacity $\bar{C}_{\mathcal{R}}(K)$ as a function of the transition probability $p_{0 \mid 0}^{1}$ of route $r_{1}$ for different values of the routing update parameter $K$, when the PU activities are modeled as Markov Chains. Specifically, the optimal route set $\mathcal{R}_{\text {opt }}=\left\{r_{1}, r_{2}\right\}$ is constructed according to the optimal priority rule (8), whereas the route set $\mathcal{R}^{\prime}=\left\{r_{2}, r_{1}\right\}$ is constructed by maximizing the route capacities $\left\{C_{i}\right\}_{i=1,2}$. We have considered three different values of the routing update parameter $K$, i.e., $K=3, K=7$, $K=14$, to confer generality to the analysis. The results confirm the theoretical analysis developed in Section III. More in detail, the average aggregate route capacity $\bar{C}_{\mathcal{R}}(K)$ is maximized by ordering the routes according to the optimal priority rule for all the considered values of the routing update period $K$. Moreover, we observe that the average aggregate route capacity $\bar{C}_{\mathcal{R}}(K)$ is deeply affected by the routing update period $K$. In fact, the higher is $K$, the higher is $\bar{C}_{\mathcal{R}}(K)$, independently of the adopted route priority rule. This is due to the inverse proportionality between the route overhead $\Omega_{m}(K)$ and the routing update parameter $K$.

In Figure 4, we report the average aggregate route capacity $\bar{C}_{\mathcal{R}}(K)$ as a function of the off probability $p_{\text {off }}^{1}$ of route $r_{1}$ for values of the routing update parameter $K$, when the PU activities are modeled as Bernoulli processes. Specifically, the optimal route set $\mathcal{R}_{\mathrm{opt}}=\left\{r_{1}, r_{2}\right\}$ is constructed according to the optimal priority rule (9), whereas the route set $\mathcal{R}^{\prime}=$ $\left\{r_{2}, r_{1}\right\}$ is constructed by maximizing the route capacities $\left\{C_{i}\right\}_{i=1,2}$. We have considered three different values of the routing update period $K$, i.e., $K=3, K=7, K=14$, to confer generality to the analysis. All the considerations we made for the previous experiment continue to hold.

\section{CONCLUSIONS}

In Cognitive Radio Networks the communication opportunities provided by a route are deeply affected by the primaryuser activities. Furthermore, whenever the route selection exploits proactively acquired information on the primaryuser activities, routing update packets need to be periodically exchanged among the nodes. The time interval between these exchanges, i.e., the routing update period, deeply affects the overall communication opportunities provided by a route, regardless of the adopted routing protocol. In this paper, we analytically derived the optimal route priority rule in Cognitive Radio Networks, by accounting for both the primary-user activity and the routing update period with the objective of maximizing the capacity available at an arbitrary Cognitive Radio user acting as source. The theoretical analysis has been conducted by adopting two different widely-adopted primaryuser activity models to confer generality to the analysis. Numerical evaluations proved the optimality of the proposed route priority rule.

\section{REFERENCES}

[1] Z. Wangt, J. Ansari, V. Atanasovski, D. Denkovski, T. Farnham, L. Gavrilovska, A. Gefflaut, R. Manfrin, E. Meshkova, J. Nasreddine, K. Rerkrai, M. Sooriyabandara, and A. Zanella, "Self-organizing home networking based on cognitive radio technologies," in IEEE Symposium on New Frontiers in Dynamic Spectrum Access Networks (DySPAN), May 2011.

[2] P. Vlacheas, R. Giaffreda, V. Stavroulaki, D. Kelaidonis, V. Foteinos, G. Poulios, P. Demestichas, A. Somov, A. Biswas, and K. Moessner, "Enabling smart cities through a cognitive management framework for the internet of things," IEEE Communications Magazine, vol. 51, no. 6, pp. 102-111, June 2013.

[3] I. F. Akyildiz, W.-Y. Lee, and K. R. Chowdhury, "Crahns: Cognitive radio ad hoc networks," Ad Hoc Networks (Elsevier), vol. 7, no. 5, pp. 810 - 836, May 2009.

[4] A. S. Cacciapuoti, M. Caleffi, and L. Paura, "A theoretical model for opportunistic routing in ad hoc networks," in International Conference on Ultra Modern Telecommunications Workshops, Oct 2009, pp. 1-7.

[5] — "Optimal constrained candidate selection for opportunistic routing," in IEEE Global Telecommunications Conference (GLOBECOM), Dec 2010, pp. 1-5.

[6] M. Cesana, F. Cuomo, and E. Ekici, "Routing in cognitive radio networks: Challenges and solutions," Ad Hoc Networks (Elsevier) Journal, vol. 9, no. 3, pp. $228-248,2011$.

[7] A. S. Cacciapuoti, M. Caleffi, F. Marino, and L. Paura, "Routing update period in cognitive radio ad hoc networks," in Proc. of IEEE International Workshop on Measurements and Networking, Oct 2013, pp. 100-104.

[8] A. S. Cacciapuoti, M. Caleffi, L. Paura, and R. Savoia, "Decision maker approaches for cooperative spectrum sensing: Participate or not participate in sensing?" IEEE Trans. on WIreless Communications, vol. 12, no. 5, pp. 2445 - 2457, Aug. 2013.

[9] D. R. Cox, Renewal Theory. New York: John Wiley and Sons Inc, 1962.

[10] H. Kim and K. Shin, "Efficient discovery of spectrum opportunities with mac-layer sensing in cognitive radio networks," IEEE Transactions on Mobile Computing, vol. 7, no. 5, pp. 533-545, 2008.

[11] A. S. Cacciapuoti, M. Caleffi, F. Marino, and L. Paura, "Optimal route priority in cognitive radio networks," under review. 